\title{
Genetic analysis of feet and leg conformation traits in Nelore cattle ${ }^{1}$
}

\author{
G. Vargas, ${ }^{2}$ H. H. R. Neves, $\dagger$ V. Cardoso,$\dagger$ D. P. Munari, $*+$ and R. Carvalheiro* $*$ \\ *Departamento de Zootecnia, Faculdade de Ciências Agrárias e Veterinárias, Universidade Estadual Paulista (Unesp), \\ Câmpus de Jaboticabal, CEP 14884-900, Jaboticabal, SP, Brazil; †Gensys Associated Consultants, CEP 90680-000, Porto \\ Alegre, RS, Brazil; and \$Conselho Nacional de Desenvolvimento Científico e Tecnológico, CEP 71605-001, Brasilia, Brazil
}

\begin{abstract}
Feet and leg conformation scores are important traits in beef cattle because they encompass a wide range of locomotion disorders that can lead to productive and reproductive losses. Thus, the study of feet and legs in beef cattle is essential for evaluating possible responses to selection focusing on minimizing economic losses caused by the occurrence of feet and leg problems. The aim of this study was to estimate variance components for feet and leg conformation traits in Nelore cattle. The data set contained records of approximately 300,000 animals that were born between 2000 and 2013. These animals belonged to the commercial beef cattle breeding program of the CRV Lagoa (www. crvlagoa.com.br). Feet and legs were evaluated by assigning visual scores at 2 different time points: feet and leg evaluated as a binary trait (FL1), measured at yearling (about $550 \mathrm{~d}$ of age) to identify whether (or not) an animal has feet and leg defects, and feet and leg score (FL2), ranging from 1 (less desirable) to 5 (more desirable) was assigned to the top $20 \%$ of animals according
\end{abstract}

to the selection index adopted by the beef cattle breeding program, which was measured 2 to 5 mo after the yearling evaluation. The FL1 and FL2 traits were analyzed together with yearling weight (YW). The (co)variance components and breeding values were estimated by Bayesian inference using 2-trait animal models. The posterior means (standard errors) of the heritabilities for FL1, FL2, and YW were 0.18 (0.04), 0.39 (0.07), and $0.47(0.01)$, respectively. The results indicate that the incidence of feet and leg problems in this population might be reduced by selection. The genetic correlation between FL1 and FL2 (-0.47) was moderate and negative as expected because the classification score that holds up each trait has opposite numerical values. The genetic trends estimated for FL1 and FL2 (-0.042 and 0.021 genetic standard deviations per year, respectively) were favorable and they indicate that the independent culling strategy for feet and leg problems promotes favorable changes and contributes to the genetic progress of these traits in the population under study.

Key words: Bayesian inference, feet and legs, genetic correlation, heritability, Nelore cattle

(C) 2017 American Society of Animal Science. All rights reserved. J. Anim. Sci. 2017.95:2379-2384 doi:10.2527/jas2016.1327

\section{INTRODUCTION}

Conformation traits such as those relating to the feet and leg of the animals have been gaining attention by many animal breeders and breeding programs due to their influence on production and profitability.

\footnotetext{
${ }^{1}$ This work was supported by Fundação de Amparo à Pesquisa do Estado de São Paulo (FAPESP), Brazil (grant number 2013/253125); and Coordenação de Aperfeiçoamento de Pessoal de Nível Superior (CAPES), Brazil (grant number 303606/2009-6).

${ }^{2}$ Corresponding author: rcar@fcav.unesp.br

Received December 20, 2016.

Accepted March 19, 2017.
}

Previous studies with beef and dairy cattle have demonstrated moderate to high levels of genetic control for feet and leg conformation traits which suggests relevant opportunity for improvement via selection (Häggman et al., 2012; Jeyaruban et al., 2012; Zavadilová and Štípková, 2012).

Brazilian breeding programs have established a system to routinely evaluate feet and leg structure by visual score in Nelore cattle and other breeds. Nowadays, some breeding programs use feet and legs as an independent culling criterion in which animals that phenotypically present these problems are discarded. However, this strategy can lead to remarkable economic losses because some of the culled 
animals have great genetic merit for other traits of economic importance (e.g., growth and carcass traits). A more efficient approach for reducing the incidence of feet and leg problems, in addition to independent culling, would be to carry out genetic evaluations of these traits and use the expected breeding values as selection criteria, highlighting the importance of studying the magnitude of genetic variability and heritability of the trait, as well as possible genetic associations with other traits undergoing selection.

Most recently, studies have been using feet and leg conformation traits to estimate heritability and correlation as well as reported economic losses, mainly in dairy cattle due to treatment dispensed and the involuntary culling of cows (Kougioumtzis et al., 2014; Kern et al., 2015). However, few published studies have examined these traits on beef cattle production, and more research in this area is necessary to find possible solutions that might reduce the incidence of feet and leg problems in the herds. The objective of this study was to estimate variance components and genetic trends for 2 traits related to feet and legs in Nelore cattle and to evaluate their association with yearling weight using data from a commercial breeding program.

\section{MATERIAL AND METHODS}

Animal Care and Use Committee approval was not necessary for this study because data were obtained from an existing database of Nelore cattle.

\section{Data}

Phenotypic records for feet and legs and pedigree information were obtained from Nelore cattle from PAINT, the beef cattle breeding program of CRV Lagoa (www.crvlagoa.com.br). The animals were raised in tropical pasture systems and belonged to herds located in Brazil and Paraguay, totaling approximately 300,000 animals with their own records for yearling weight collected from 2000 to 2013.

Feet and legs were evaluated by trained technicians of PAINT, which assigned visual scores to the overall structure of feet and legs at 2 different time points. Two different traits were defined: feet and leg evaluated as a binary trait (FL1), measured at yearling (about $550 \mathrm{~d}$ of age), to identify whether $(\mathrm{FL} 1=1)$ or not $(\mathrm{FL} 1=0)$ an animal had feet and leg problems; and feet and leg score (FL2), ranging from 1 (less desirable) to 5 (more desirable), was assigned to the top $20 \%$ of animals according to the selection index adopted by the breeding program. The selection index considers EPD for the following traits: birth to weaning weight gain, visual scores of conformation, finishing precocity, muscling, navel/prepuce at weaning and yearling, weaning to yearling weight gain, temperament at yearling, and scrotal circumference. These animals were candidates to receive the Special Certificate of Identification and Production (CEIP), an official certificate that testifies the value of seedstock delivered by breeding programs, i.e., animals that are genetically classified as superior (top 20\%; Horimoto et al., 2007).

In the breeding program considered in this study, animals that received score 1 for FL1 could not be candidates to receive the CEIP, and therefore were not evaluated for FL2. The evaluation of FL2 occurred a few days after the animals were evaluated at yearling, when the results of routine genetic evaluations were released and CEIP candidates were defined. Furthermore, animals with an undesirable score for FL2 (FL2 = 1) were not allowed to receive a CEIP certificate, a procedure that often results in economic losses because genetically superior animals for growth and carcass traits have to be culled.

\section{Statistical Methods}

In preliminary analyses, generalized linear mixed models were fitted using the lme4 package of the $\mathrm{R}$ software (R Core Team, 2013) to identify environmental effects that influenced FL1 and FL2. Based on the results of these analyses, the final model for the genetic evaluation of the two traits included the fixed effect of contemporary group (CG) and the linear effect of yearling age as covariate. The CG was defined based on the concatenation of the effects of herd, year and season of birth, management group at weaning, date of measurement at yearling, and management group at yearling. The sex of the animal was not included in the definition of CG because the management groups consisted of animals of the same sex. After analyzing the data consistency, CG considered disconnected from the main database (connected groups) were excluded. Genetic connectedness among CG was verified using the AMC software (Roso and Schenkel, 2006), which evaluates connectedness among CG on the basis of total direct genetic links between them due to common sires, common sires and dams, or any common ancestor, in a way that genetic links are weighted by the corresponding additive relationships. A minimum of 10 direct genetic links were required to include a $\mathrm{CG}$ in the main set of connected groups.

Bayesian inference was used to estimate the variance components and predict the breeding values. Three different 2-trait analyses were performed for FL1, FL2, and yearling weight (YW), adjusting a threshold-threshold (FL1-FL2) or a linear-threshold (YW-FL1; YW-FL2) animal model with the THRGIBBS1F90 software (Misztal, 
2014). The analyses considering YW aim to evaluate possible correlated responses in feet and legs and to alleviate the effect of sequential selection in the analyses of FL2 (Pollak and Quaas, 1981), since just part of the animals evaluated at yearling are also evaluated for FL2.

\section{Two-Trait Model}

The statistical model used in the different analyses can be written in matrix notation as

$$
\mathbf{y}_{i}=\mathbf{X} \boldsymbol{\beta}_{i}+\mathbf{Z a}_{i}+\mathbf{e}_{i},
$$

where $\mathbf{y}_{i}$ is a vector of observations (or liabilities, in the case of FL1 and FL2) for the $i$ th trait $(i=1,2) ; \boldsymbol{\beta}_{i}$ is a vector of systematic effects (CG and the linear effect of yearling age); $\mathbf{a}_{i}$ is a vector of additive direct genetic effects; $\mathbf{e}_{i}$ is a vector of random residual effects, and $\mathbf{X}$ and $\mathbf{Z}$ are incidence matrices that relate the phenotypic information of the $i$ th trait to effects $\boldsymbol{\beta}_{i}$ and $\mathbf{a}_{i}$, respectively. For feet and legs (FL1 and FL2), it is expected that the inclusion of the $\mathrm{CG}$ effect in the model accounts for part of the environmental effects and corrects for the effect of evaluator, given that a single technician evaluates all animals of the same management group.

The assumptions of model (1) can be described as follows:

$$
\mathbf{E}\left[\begin{array}{c}
\mathbf{y}_{\mathrm{i}} \\
\mathbf{a}_{\mathrm{i}} \\
\mathbf{e}_{\mathrm{i}}
\end{array}\right]=\left[\begin{array}{c}
\mathbf{X} \boldsymbol{\beta}_{i} \\
0 \\
0
\end{array}\right] .
$$

The covariance structure between the random effects in (1) can be described as follows:

$$
\operatorname{Var}\left[\begin{array}{l}
\mathbf{a} \\
\mathbf{e}
\end{array}\right]=\left[\begin{array}{cc}
\mathbf{A} \otimes \boldsymbol{\Sigma}_{\mathrm{a}} & 0 \\
0 & \mathbf{I}_{N} \otimes \boldsymbol{\Sigma}_{e}
\end{array}\right],
$$

where $\boldsymbol{\Sigma}_{a}$ is the additive direct genetic covariance matrix; $\boldsymbol{\Sigma}_{e}$ is the residual covariance matrix; $\mathbf{A}$ is the numerator relationship matrix; $\otimes$ denotes the direct product between matrices; $\mathbf{I}$ is an identity matrix, and $N$ is the number of animals. Since there was no variation in FL1 within levels of FL2 (i.e., FL1 $=0$ for all animals evaluated for FL2), the residual covariance between FL1 and FL2 would not be estimable and was therefore assumed to be zero.

In the analysis of FL1, considering that the variable in the underlying distribution is not observable, the parameterization $\sigma_{\mathrm{e}}^{2}=1$ was adopted to allow identification of the variable in the likelihood function (Gianola and Sorensen, 2002). In the case of FL2, according to Sorensen et al. (1995), the thresholds $\mathrm{t} 1$ and $\mathrm{t} 2$ were kept fixed at 0 and 1 , respectively, so that $\sigma_{\mathrm{e}}^{2}$ could be estimated.

\section{Threshold Model}

A threshold model was assumed for FL1 and FL2. Threshold models postulate that categorical traits are determined by nonobservable continuous variables in an underlying scale (hereafter liabilities), so that a set of (j-1) thresholds correspond to the discontinuities in the observable scale for $j$ mutually exclusive ordered categories. Thus, initial threshold values are fixed as follows: $\mathrm{t}_{1}<\mathrm{t}_{2}<\ldots<\mathrm{t}_{\mathrm{j}-1}$, assuming that $\mathrm{t}_{0}=-\mathrm{Y}$ and $\mathrm{t}_{\mathrm{j}}=+\mathrm{Y}$ (Gianola and Foulley, 1983). In the case of FL1 and FL2, it was assumed that the categories or scores for each animal $i$ were defined by the liability $U_{i}$ in the underlying scale as follows:

$$
\begin{aligned}
& \text { FL1: }\left\{\mathrm{FL}_{\mathrm{i}}=0 \text { if } \mathrm{U}_{\mathrm{i}} \leq \mathrm{t} 1\right. \\
& \left.\mathrm{FL}_{\mathrm{i}}=1 \text { if } \mathrm{U}_{\mathrm{i}}>\mathrm{t} 1\right\} \\
& \text { FL2: }\left\{\text { FL2 } \mathrm{i}=1 \text { if } \mathrm{U}_{\mathrm{i}} \leq \mathrm{t} 1\right. \text {; } \\
& \mathrm{FL}_{\mathrm{i}}=2 \text { if } \mathrm{t} 1<\mathrm{U}_{\mathrm{i}} \leq \mathrm{t} 2 \text {; } \\
& \mathrm{FL}_{2}=3 \text { if } \mathrm{t} 2<\mathrm{U}_{\mathrm{i}} \leq \mathrm{t} 3 \text {; } \\
& \mathrm{FL}_{\mathrm{i}}=4 \text { if } \mathrm{t} 3<\mathrm{U}_{\mathrm{i}} \leq \mathrm{t} 4 \text {; } \\
& \left.\mathrm{FL}_{\mathrm{i}}=5 \text { if } \mathrm{t} 4<\mathrm{U}_{\mathrm{i}} \leq \mathrm{t} 5\right\} .
\end{aligned}
$$

\section{Bayesian Analysis}

A single chain of $1,000,000$ cycles was generated for each analysis, applying a conservative burnin period of 100,000 cycles and a thinning interval of 250 cycles. The coda package of the $\mathrm{R}$ software ( $\mathrm{R}$ Core Team, 2013) was used to assess the convergence of chains through visual inspection and with the Geweke (Geweke, 1992) and Heidelberger and Welch (Heidelberger and Welch, 1983) tests.

Genetic trends were estimated by linear regression of the posterior means of estimated breeding values for each trait on the year of birth. The posterior means of estimated breeding values were standardized in terms of the posterior mean of the genetic standard deviation. Linear regression analysis using individual inbreeding coefficients of the animals as covariable and their corresponding estimated breeding values as response variable were performed attempting to identify possible effect of inbreeding on FL1 and FL2.

\section{RESULTS AND DISCUSSION}

For FL2, most of the animals were classified as score 3 to $5(71.2 \%$; Table 1$)$, suggesting satisfactory feet and leg structure in the majority of animals. The incidence of animals presenting defective feet and legs was about 5\% 
Table 1. Summary statistics of feet and legs in Nelore cattle

\begin{tabular}{|c|c|c|c|c|c|c|c|c|c|c|}
\hline \multirow[b]{2}{*}{ Traits 1} & \multicolumn{4}{|c|}{ Data structure $^{2}$} & \multicolumn{6}{|c|}{ Score frequency distribution ${ }^{3}$} \\
\hline & $\overline{\mathrm{N}}$ & NCG & NS & ND & 0 & 1 & 2 & 3 & 4 & 5 \\
\hline \multirow[t]{2}{*}{$\overline{\text { FL1 }}$} & 96,836 & 2105 & 748 & 73,272 & 92,469 & 4367 & - & - & - & - \\
\hline & & & & & $(95.5)$ & $(4.5)$ & - & - & - & - \\
\hline \multirow[t]{2}{*}{ FL2 } & 14,708 & 897 & 340 & 12,920 & - & 1040 & 3192 & 6088 & 3616 & 772 \\
\hline & & & & & - & (7.1) & (21.7) & (41.4) & (24.6) & (5.2) \\
\hline
\end{tabular}

${ }^{1} \mathrm{FL} 1$ = feet and leg evaluated as a binary trait (scores assigned to all animals measured at yearling); FL2 = feet and leg scores ranging from 1 (less desirable) to 5 (more desirable), assigned to the top $20 \%$ animals according to the selection index applied to this population.

${ }^{2} N=$ number of observations; $\mathrm{NCG}=$ number of contemporary groups; $\mathrm{NS}=$ number of sires; $\mathrm{ND}=$ number of dams.

${ }^{3}$ The absolute frequency of each score is given, followed by the relative proportion (in \%).

$(4,367 / 96,836)$ for FL1 and 7\% $(1,040 / 14,708)$ for FL2 (Table 1), indicating the possible occurrence of considerable economic losses because these animals are generally unable to perform their productive and reproductive functions satisfactorily due to these problems.

The average estimates of direct heritability and genetic correlations for FL1, FL2, and YW obtained by 2-trait analyses are shown in Table 2 . The heritability estimate of FL1 was lower than the FL2 (posterior means of 0.18 and 0.39 , respectively) and greater than reported by Lima et al. (2013) and Sima (2015; 0.06 and 0.07, respectively) in Nelore cattle. Jeyaruban et al. (2012) evaluated 6 different types of feet and leg conformation traits in Australian Angus and reported heritability estimates ranging from 0.22 to 0.50 . This result may be explained by the lack of systematic routine evaluation of feet and legs at yearling so that only extreme cases are notified. In addition, the dichotomization of feet and leg evaluation in FL1 may cause some loss of information when compared to FL2, resulting in smaller variability. For polychotomous traits, a similar type of dichotomization has been associated with loss of power in genetic studies (Brisbin et al., 2010). Another factor that might explain the differences between the heritability estimates for FL1 and FL2 is the lower reproducibility of feet and leg assessment at yearling (assessment scores ranging from 0 to 1 to identify whether or not an animal had feet and leg problems) when compared to the more detailed evaluations performed for FL2 (assessment scores ranging from 1 to 5).

The heritability and additive genetic variances obtained for FL2 (0.39 and 0.07) were similar to those reported by Passafaro et al. (2013; 0.36 and 0.08$)$, who analyzed data from a different Nelore population using a slightly different evaluation system (scores ranging from 1 to 6 ). These findings suggest that feet and legs should respond to selection. Considering the larger number of evaluation categories for FL2 compared to FL1, the former is theoretically more appropriate to describe the variability in feet and legs between animals. A disadvantage of FL2 is that it is evaluated only in part of the yearling animals and is possibly more prone to bias due to preselection. An alternative would be to treat both, FL1 and FL2, as complementary traits in routine genetic evaluations.

The genetic correlation between FL1 and FL2 $(-0.47)$ was negative and had a moderate magnitude. The sign of this estimate is expected considering that, for FL1, the lower classification score (0) suggests that the animal does not have defective feet or legs, while in the case of FL2 animals with better feet and legs receive the greatest score. The factors cited above as an explanation for the difference between the heritability estimates for FL1 and FL2 may also explain why the genetic correlation between FL1 and FL2 was not stronger. The results of genetic correlations were similar to those observed in previously published studies in dairy cattle (Berry et al., 2004; Bohlouli et al., 2015). Bohlouli et al. (2015) reported genetic correlations between rear leg rear view and foot angle of -0.67 , and between rear leg rear view and rear leg side view of -0.11 . The greatest absolute value has been reported by Berry et al. (2004), who obtained a genetic correlation of -0.88 between leg position and hoof angle. However, estimates of genetic correlations from different studies cannot be directly comparable because of differences in data sets, models, type of feet and leg conformation traits analyzed, and the scores considered to evaluate the traits.

The genetic correlation between YW and feet and legs had low (0.04) and moderate magnitude (0.39) to FL1 and FL2, respectively. In dairy cattle, previous studies have reported important influence of the body weight on the incidence of some diseases in feet, resulting in locomotion problems (Neveux et al., 2006; Van Nuffel et al., 2015). According to the studies, a lower incidence of this problem can be observed when the body weight is evenly distributed between the animal limbs so that the foot and leg maintains normal conformation, reducing stress on ligaments, tendons, and joints, as well mechanical defects, resulting in physical incapability. Using information of Holstein cows, Campos et al. (2012) reported genetic 
Table 2. Heritability estimates $\left(\mathrm{h}^{2}\right)$ for FL1, FL2, and YW obtained in 2-trait analyses (diagonal), genetic (above the diagonal), and environmental (below diagonal) correlations*, and their standard deviation (in parentheses) for Nelore cattle

\begin{tabular}{lccc}
\hline \hline Traits $^{1}$ & FL1 & FL2 & YW \\
FL1 & $0.18(0.04)$ & $-0.47(0.02)$ & $0.04(0.07)$ \\
FL2 & - & $0.39(0.07)$ & $0.39(0.06)$ \\
YW & $-0.19(0.03)$ & $0.11(0.03)$ & $0.47(0.01)$ \\
\hline
\end{tabular}

*Estimates obtained as the average of the marginal posterior distribution of the parameters.

${ }^{1} \mathrm{FL} 1=$ feet and legs evaluated as a binary trait (scores assigned to all animals measured at yearling); FL2 = feet and leg scores ranging from 1 (less desirable) to 5 (more desirable), assigned to the top 20\% animals according to the selection index applied to this population; $\mathrm{YW}=$ yearling weight (measured at about $550 \mathrm{~d}$ ).

correlations between body weight and traits related to feet and legs, ranging from 0.01 to 0.28 .

The environmental correlations of YW with FL1 $(-0.19)$ and FL2 (0.11) were of low magnitude. The residual correlation between FL1 and FL2 was not estimated because animals with some types of feet and leg defects, i.e., those classified as score 1 for FL1, were not candidates to receive the certificate of genetic superiority and were therefore not evaluated for FL2.

The genetic trends for FL1, FL2, and YW are shown in Fig. 1. The coefficients of determination of the models used to regress the average breeding values for FL1, FL2, and YW on an animal's year of birth were all greater than 0.69 , indicating a reasonable fit of the linear regression. Genetic trends of -0.042 and 0.021 standard deviations per year were obtained for FL1 and FL2, respectively. Although relatively low, the genetic trends for FL1 and FL2 occurred in a desirable direction. This result suggests that the independent culling strategy for feet and leg problems adopted by the breeding program is causing favorable changes, contributing to the genetic progress of the population. The genetic trend for FL2 may also be due to an indirect response to selection because YW was positively correlated with FL2 and showed a favorable genetic trend (Fig. 1). No genetic trends for feet and leg traits were found in the literature to be used as comparisons.

Finally, analysis of the association between individual inbreeding coefficients of the animals and their corresponding estimated breeding values revealed no significant associations (data not shown). However, it is not clear to what extent this result is related to the lack of influence of inbreeding on feet and leg problems or to the low quality of the available pedigree information because roughly half the calves controlled in this breeding program have unknown sires (use of multiple sire mating).
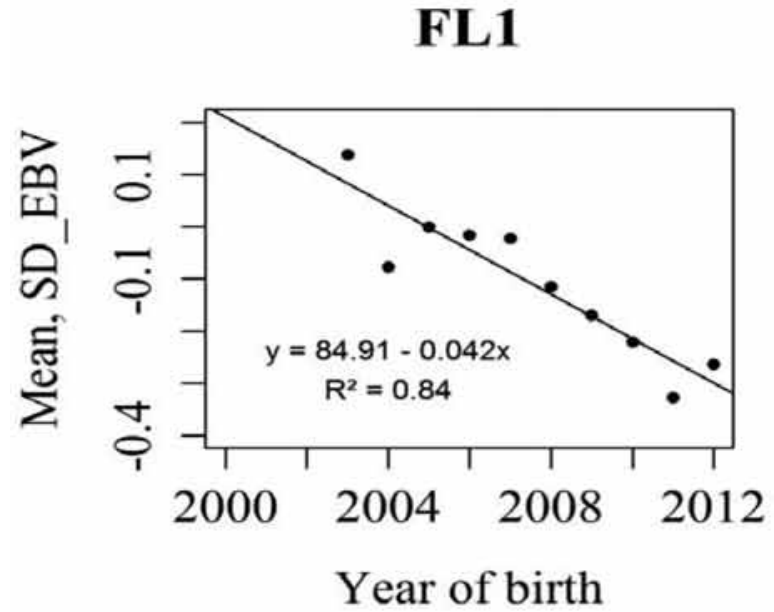

FL2

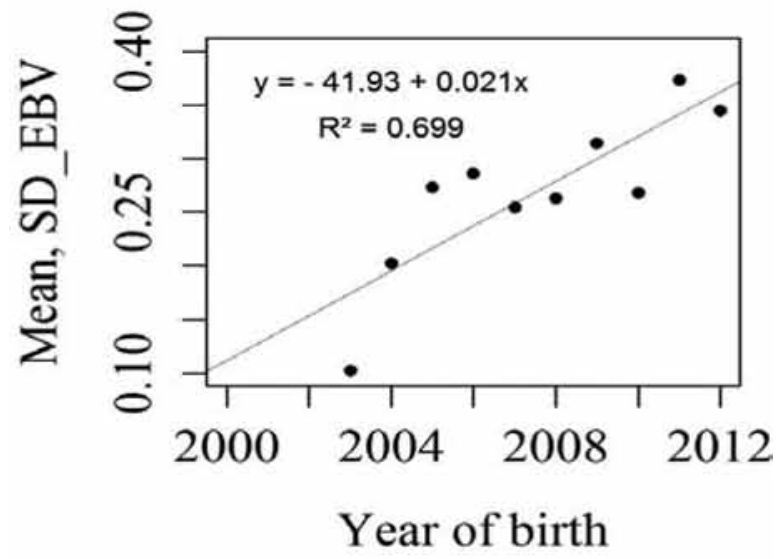

YW

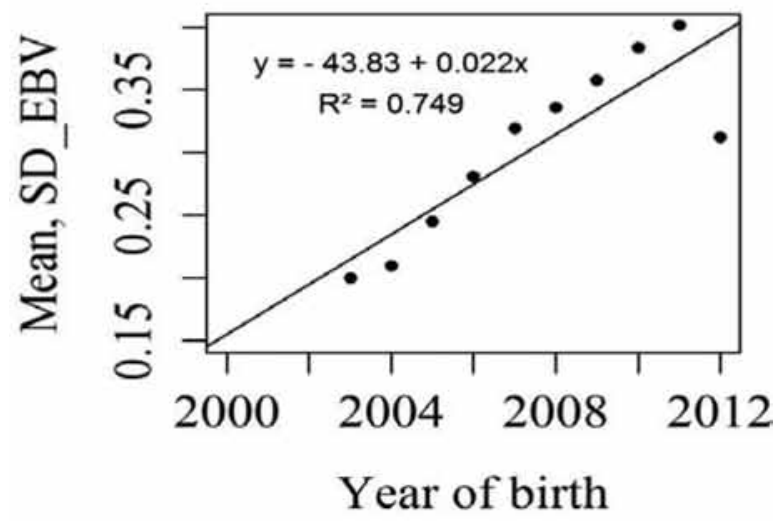

Figure 1. Genetic trends estimated by linear regression of the posterior means of estimated breeding values for FL1, FL2, and YW in a population of Nelore cattle. FL1 = feet and legs evaluated as a binary trait measured at yearling to identify whether (1) or not (0) an animal had feet and leg problems; FL2 = feet and leg scores ranging from 1 (less desirable) to 5 (more desirable), assigned to the top $20 \%$ of animals according to the selection index applied to this population, which consists of productive and reproductive traits measured at weaning and yearling; $\mathrm{YW}=$ yearling weight (measured at about $550 \mathrm{~d}$ ). Mean, SD EBV = posterior means of estimated breeding value (EBV) expressed as standard deviation (SD). 


\section{LITERATURE CITED}

Berry, D. P., F. Buckley, P. Dillon, D. R. Evans, and R. F. Veerkamp. 2004. Genetic relationships among linear type traits, milk yield, body weight, fertility and somatic cell count in primiparous dairy cows. Ir. J. Agric. Food Res. 43:161-176.

Brisbin, A., M. M. Weissman, A. J. Fyer, S. P. Hamilton, J. A. Knowles, C. D. Bustamante, and J. G. Mezey. 2010. Bayesian linkage analysis of categorical traits for arbitrary pedigree designs. PLoS One 5:e12307.

Bohlouli, M., S. Alijani, and M. R. Varposhti. 2015. Genetic relationships among linear type traits and milk production traits of Holstein dairy cattle. Ann. Anim. Sci. 15:903-917.

Campos, R. V., J. A. Cobuci, C. N. Costa, and J. B. Neto. 2012. Genetic parameters for type traits in Holstein cows in Brazil. Rev. Bras. Zootec. 41:2150-2161.

Geweke, J. 1992. Evaluating the accuracy of sampling-based approaches to calculating posterior moments. 4th ed. Clarendon, Oxford, UK.

Gianola, D., and J. L. Foulley. 1983. Sire evaluation for ordered categorical data with a threshold model. Genet. Sel. Evol. 15:201-224.

Gianola, D., and D. Sorensen. 2002. Likelihood, Bayesian, and MCMC methods in quantitative genetics. 2nd ed. Springer, New York.

Häggman J., J. Juga, M. J. Sillanpää, and R. Thompson. 2012. Genetic parameters for claw health and feet and leg conformation traits in Finnish Ayrshire cows. J. Anim. Breed. Genet. 130:89-97.

Heidelberger, P., and P. D. Welch. 1983. Simulation Run Length Control in the Presence of an Initial Transient. Oper. Res. 31:1109-1144.

Horimoto, A. R., J. B. Ferraz, J. C. C. Balieiro, and J. P. Eler. 2007. Phenotypic and genetic correlations for body structure scores (frame) with productive traits and index for CEIP classification in Nelore beef cattle. Genet. Mol. Res. 6:188-196.

Jeyaruban, G., B. Tier, D. Johnston, and H. Graser. 2012. Genetic analysis of feet and leg traits of Australian Angus cattle using linear and threshold models. Anim. Prod. Sci. 52:1-10.

Kern, E. L., J. A. Cobuci, C. N. Costa, C. M. McManus, and J. B. Neto. 2015. Genetic association between longevity and linear type traits of Holstein cows. Sci. Agric. 72:203-209.
Kougioumtzis, A., G. E. Valergakis, and G. Oikonomou. 2014. Profile and genetic parameters of dairy cattle locomotion score and lameness across lactation. Animal 8:20-27.

Lima, P. R. M., S. R. Paiva, J. A. Cobuci, J. Braccini Neto, C. H. Machado, and C. McManus. 2013. Genetic parameters for type classification of Nelore cattle on central performance tests at pasture in Brazil. Trop. Anim. Health Prod. 45:1627-1634.

Misztal, I. 2014. Animal Breeding and Genetics Group. http://nce.ads. uga.edu/wiki/doku.php?id=application_programs. (Accessed 21 February 1999.)

Neveux, S., D. M. Weary, J. Rushen, M. A. von Keyserlingk, and A. M. de Passillé. 2006. Hoof discomfort changes how dairy cattle distribute their body weight. J. Dairy Sci. 89:2503-2509.

Van Nuffel, A., I. Zwertvaegher, L. Pluym, S. Van Weyenberg, V. M. Thorup, M. Pastell, B. Sonck, and W. Saeys. 2015. Lameness detection in dairy cows: Part 1 . How to distinguish between non-lame and lame cows based on differences in locomotion or behavior. Animals (Basel) 5:838-860.

Passafaro, T. L., F. S. S. Raidan, M. P. Aquino, T. C. Escarce, L. A. Josahkian, and F. L. B. Toral. 2013. Parâmetros genéticos para escores visuais de tourinhos Nelore com modelos lineares e de limiar. In: Proc. 10th Simp. Bras. Melhor., Uberaba, Brazil. p. 3.

Pollak, E. J., and R. L. Quaas. 1981. Monte Carlo study of genetic evaluations using sequentially selected records. J. Anim. Sci. 52:257-264.

R Core Team. 2013. R: A language and environment for statistical computing. R Found. Stat. Comput., Vienna.

Roso, V. M., and F. Schenkel. 2006. A computer program to assess the degree of connectedness among contemporary groups. In: Proc. 8th World Congr. Genet. Appl. Livest. Prod. XIII, Belo Horizonte, Brazil. p. 27-26.

Sima, P. S. 2015. Statistical models for genetic analysis visual scores in beef cattle. PhD Diss. Belo Horizonte, Brazil.

Sorensen, D. A., S. Andersen, D. Gianola, and I. Korsgaard. 1995. Bayesian inference in threshold models using Gibbs sampling. Genet. Sel. Evol. 27:229-249.

Zavadilová, L., and M. Štípková. 2012. Genetic correlations between longevity and conformation traits in the Czech Holstein population. J. Anim. Sci. 57:125-136. 
Reproduced with permission of copyright owner.

Further reproduction prohibited without permission. 